\title{
Atomic study of Hybrid Spintronic Heterostructures: $\mathrm{Co}_{2} \mathrm{FeAl}_{0.5} \mathrm{Si}_{0.5} / \mathrm{Ge}(111)$
}

Z Nedelkoski ${ }^{1}$, D.M. Kepaptsoglou ${ }^{2}$, B. Keurbanjiang ${ }^{1}$, Q.M. Ramasse ${ }^{2}$, A. Ghasemi ${ }^{1}$, C. Love ${ }^{1}$, S. Cavill $^{1}$, K. Hamaya ${ }^{4}$, S. P. Tear ${ }^{1,3}$, and V.K. Lazarov ${ }^{1}$

${ }^{1}$ Department of Physics, University of York, Heslington, York, YO10 5DD, UK,

${ }^{2}$ SuperSTEM Laboratory, STFC Daresbury Campus, Warrington, WA4 4AD, UK

${ }^{3}$ York JEOL Nanocentre, University of York, Heslington, York, YO10 5BR, UK

${ }^{4}$ Department of Systems Innovation, Osaka University, Osaka 560-8531, Japan

Engineering of the atomic structure of hetero-interfaces enables tuning of electronic properties of the heterostructure such as band alignment, Schottky barrier height, interface conductivity and magnetism. Hence it is the aim of continuous experimental and theoretical research to control chemical intermixing arising due to interdiffusion, as well as interface strain, which are the main parameters that control the structural and chemical quality of a given heterostructure. However, tailoring the atomic structure of ferromagnet/semiconductor interfaces, of crucial importance for spintronic applications, has been shown to be a rather elusive goal despite the intensive research efforts over the past years $[1,2]$.

In this work, in the case of half metallic $\mathrm{Co}_{2} \mathrm{FeAl}_{0.5} \mathrm{Si}_{0.5}$ (CFAS) and Ge we demonstrate that atomically sharp half metal/semiconductor interfaces are achievable with almost no strain due to the excellent lattice match between CFAS and Ge. We show that the film has the desirable B2 ordering which provides high spin polarisation and it does not form any secondary phases in the interface region. Based on state-of-the-art aberration-corrected high angle annular dark field (HAADF) scanning transmission electron microscopy (STEM) imaging we have determined that the atomic structure at the interface CFAS/Ge(111) interface is realised via Ge-Co bonds, Fig. 1.

Based on atomistic models derived from HAADF-STEM we construct a realistic interface model. Density functional theory calculations show that this interface atomic structure preserves both the high spin-polarization of the CFAS film as well as its magnetic moment in the interface vicinity, making this system an excellent platform for spin-based device applications, Fig. 2.The atomic level spectroscopic studies were employed to investigate the chemical abruptness of the studied interface. Atomic resolution electron energy loss spectroscopy chemical mapping reveal small and selective out-diffusion of Ge within a $\sim 1 \mathrm{~nm}$ region of the interface [3]. This atomic plane selective diffusion process does not change the structural integrity and spin-electronic structure of the CFAS since the outdiffused Ge selectively substitutes only $\mathrm{Fe}$ and $\mathrm{Si} / \mathrm{Al}$ atoms, which in turn does not affect the film's half-metallicity.

Finally we report on the annealing effect on the film structures and overall magnetic properties of the CFAS thin films on Ge. Ferromagnetic resonance and vibrational magnetometry shows that mild annealing temperatures improve the magnetic properties i.e. increase the saturation magnetisation and decrease the Gilbert damping constant. Annealing above $450^{\circ} \mathrm{C}$ significantly promotes Ge outdiffusion into the film, hence both structural integrity of the hetero-interface and overall magnetic properties of the film deteriorate. 


\section{References}

[1] A. Khon et al. J Appl Phys. (2007), 101, 023915.

[2] B. Kuerbanjiang et al. Appl Phys Lett. (2016), 108, 172412.

[3] Z. Nedelkoski et al. Sci Rep. (2016), 6: 37282.

[4] The authors acknowledge funding from EPSRC via research grants EP/K03278X/1. SuperSTEM is the U.K. National Facility for Aberration-Corrected STEM funded by the EPSRC.

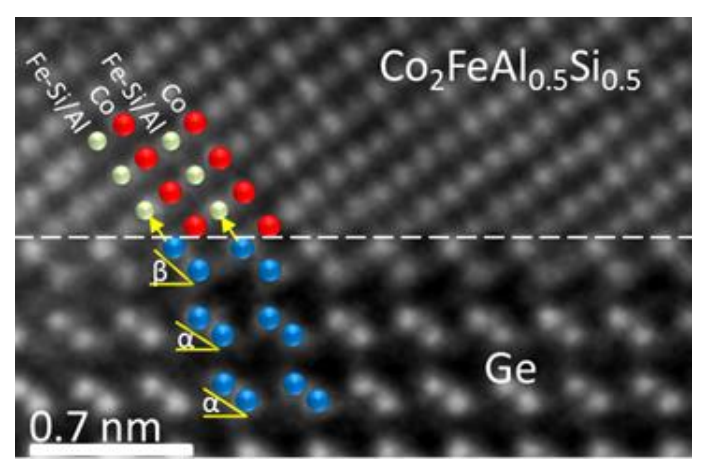

Fig. 1: Atomic resolution HAADF STEM image of the interface showing that the CFAS film terminates on a $\operatorname{Co}(111)$ atomic plane. The colour coding of the overlaid structural model is as follows: $\mathrm{Ge}$-blue; $\mathrm{Co}$-red; Fe-Si/Al -grey. The white scale-bar corresponds to $0.7 \mathrm{~nm}$. The tilt angle of the Ge dumbbells from $\alpha=37^{\circ}$ in the bulk-like region increases to $\beta=51^{\circ}$ for the interfacial Ge bilayer.

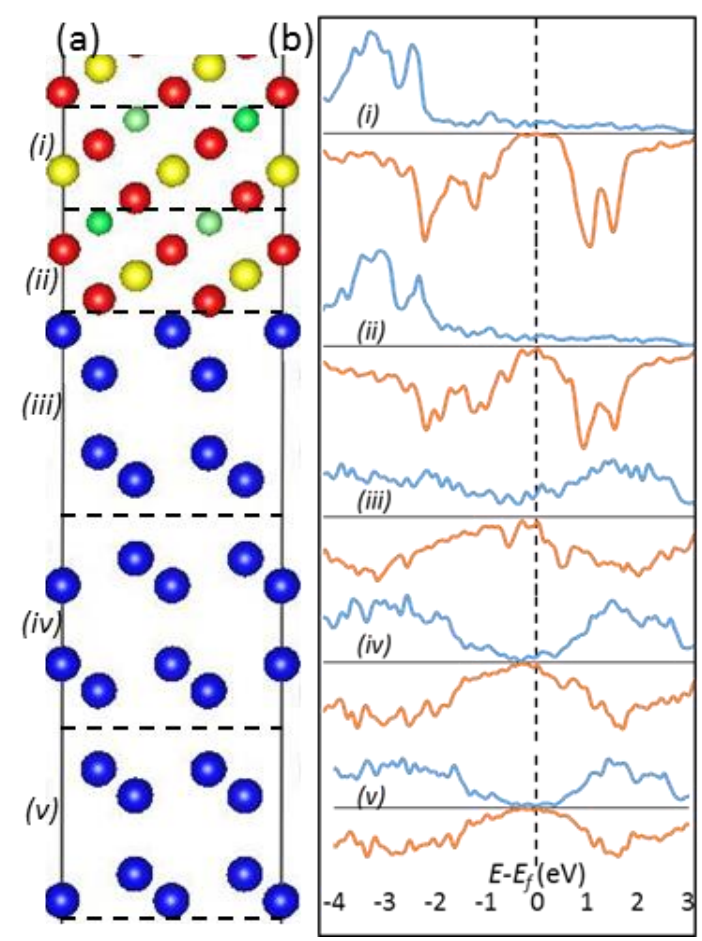

Fig. 2: a) Structural model of the CFAS/Ge interface. b) Spin-polarized partial density of states (PDOS) for the regions labelled as (i)-(v) in (a). Spin-up PDOS are presented in the upper part of the plots, while spin-down in the lower part of the plots. 